\title{
Magnetoresistance of Half-Metallic Oxide Nanocontacts
}

\author{
J. J. Versluijs, M. A. Bari, and J.M.D. Coey \\ Physics Department, Trinity College, Dublin 2, Ireland
}

(Received 16 January 2001; published 22 June 2001)

\begin{abstract}
Magnetoresistive effects $(R(0)-R(H)) / R(H)$ exceeding $500 \%$ are found at room temperature in a field of $7 \mathrm{mT}$ in nanocontacts between $\mathrm{Fe}_{3} \mathrm{O}_{4}$ crystallites. The shape of the $I(V)$ curve depends on field and the magnitude of the magnetoresistance is correlated with the resistance, the largest effects occurring when $R>100 \mathrm{k} \Omega$. The explanation proposed involves hopping transport of spin-polarized electrons through a narrow domain wall pinned at the nanocontact; spin pressure on the domain wall pushes it out into the electrode, leading to the nonlinearity of the $I(V)$ characteristic. Application of current-induced wall motion in a simple fast-switching magnetic memory element is proposed.
\end{abstract}

Conventional electronics ignores the spin of the electron. If the budding science of spin electronics is to bear fruit, devices have to be designed around effects which are large at room temperature. Half-metallic oxides are potential sources of fully spin-polarized electrons, and when they are used to inject electrons across thin tunnel barriers [1,2], grain boundaries [3], or interparticle contacts [4] large magnetoresistance effects are observed, at liquid helium temperatures. For reasons that remain uncertain, the spin polarization falls rapidly with increasing temperature and the magnetoresistance at room temperature in all-oxide structures is usually no more than $1 \%$. In this Letter, we show that the conductivity of a nanocontact between two crystallites of magnetite $\left(\mathrm{Fe}_{3} \mathrm{O}_{4}\right)$ can increase sixfold at room temperature in an applied field of $7 \mathrm{mT}$ (70 Oe). This large low-field effect is attributed to spin-polarized electrons hopping across a very narrow domain wall at the contact. Spin pressure on the domain wall at the nanocontact leads to nonlinearity of the $I(V)$ characteristic, and the possibility of fast switching in a bistable two- or threeterminal device via current-induced wall motion.

Magnetite, the ferrimagnetic inverse spinel $\mathrm{Fe}_{3} \mathrm{O}_{4}$, is the half-metallic oxide with the highest known Curie temperature $(860 \mathrm{~K})$. Carriers are small polarons in a minority spin $3 d^{\downarrow}\left(t_{2 g}\right)$ band [5] which hop among the B sites; the majority spin band is full [6]. Tiny magnetite crystals grown by chemical vapor transport are used for our experiments [7]. Two of them are glued in a simple piezoelectric device with vibration isolation that allows electrical contact to be made or broken in a controlled manner [8], following a method of Costa-Krämer et al. [9]. All measurements are carried out at room temperature $(290 \mathrm{~K})$ in ambient air. A magnetic field of up to $14 \mathrm{mT}$ (140 Oe) can be applied in any direction in a plane by means of two pairs of Helmholtz coils.

When the contact is broken rapidly in a relay $(\approx 100 \mu \mathrm{s})$, the conductance falls in an irregular way. A histogram based on 500 such breaks shows a single weak peak near the quantum of conductance $G_{0}=2 e^{2} / h=(12.9 \mathrm{k} \Omega)^{-1}$. There is much less structure than was found previously for contacts between crystallites of $\left(\mathrm{La}_{0.7} \mathrm{Sr}_{0.3}\right) \mathrm{MnO}_{3}$ [10]. In slowly broken contacts $(\approx 1 \mathrm{~s})$, however, the conductance decreases continuously to zero.

For the present experiments, we arrest the break at a contact resistance of 10-500 k $\Omega$, and measure the currentvoltage curves $I(V)$ and the magnetoresistance $I(H)$. The largest effects are measured in contacts where $G \ll G_{0}$. The contacts are remarkably stable, persisting for a minute or more. They can be reversibly cycled many times. Hence, there is no evidence for a heating effect in the range of current used. Several $I(V)$ curves are shown in Fig. 1. They are fitted as $I=G V+c V^{3}$. The more conducting the contact, the more Ohmic it appears. Examination of 30 contacts indicates that $c=G^{0.3 \pm 0.1}$, a weaker correlation than was found in $\left(\mathrm{La}_{0.7} \mathrm{Sr}_{0.3}\right) \mathrm{MnO}_{3}$ [11]. A few of the $I(V)$ curves are significantly asymmetric for positive and negative voltages.

The effect of applying a small magnetic field is always to increase the conductance of the contact. One such data set is

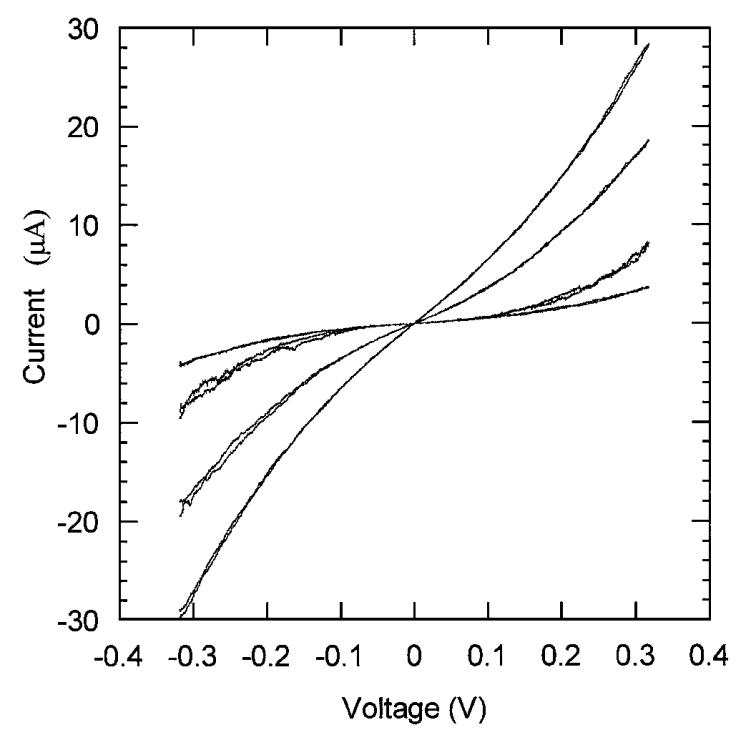

FIG. 1. $I(V)$ characteristics for some typical nanocontacts between two $\mathrm{Fe}_{3} \mathrm{O}_{4}$ crystals at $290 \mathrm{~K}$. 
shown in Fig. 2(a). The $7 \mathrm{mT}$ field in this case increases $G$ by a factor of 6.4, corresponding to a magnetoresistance $\Delta R / R(H)=540 \%$, where $\Delta R=R(0)-R(H)$. The conductance does not quite return to its initial value on cycling the field back to zero. There is some magnetic hysteresis, illustrated in Fig. 2(b). The magnetoresistance effect is independent of whether the field is applied parallel or perpendicular to the current direction. Furthermore, when a rotating field of frequency $f=110 \mathrm{~Hz}$ is applied in the plane including the current direction, no appreciable signal at $2 f$ is detected. Figure 3 summarizes the magnetoresistive effect expressed as $\Delta R / R(0)$ for measurements on many $\mathrm{Fe}_{3} \mathrm{O}_{4}$ contacts. Effects of up to $16 \%$ have also been found for contacts between $\left(\mathrm{La}_{0.7} \mathrm{Sr}_{0.3}\right) \mathrm{MnO}_{3}$ crystals. Note that with this definition of magnetoresistance, the effect cannot exceed $100 \%$.

So what can be the explanation of the huge magnetoresistance in these contacts? It is many times larger than has been found at room temperature in any other ferromagnetic oxide or metal tunnel junction structure [12,13], and it is almost double that reported for ballistic nickel
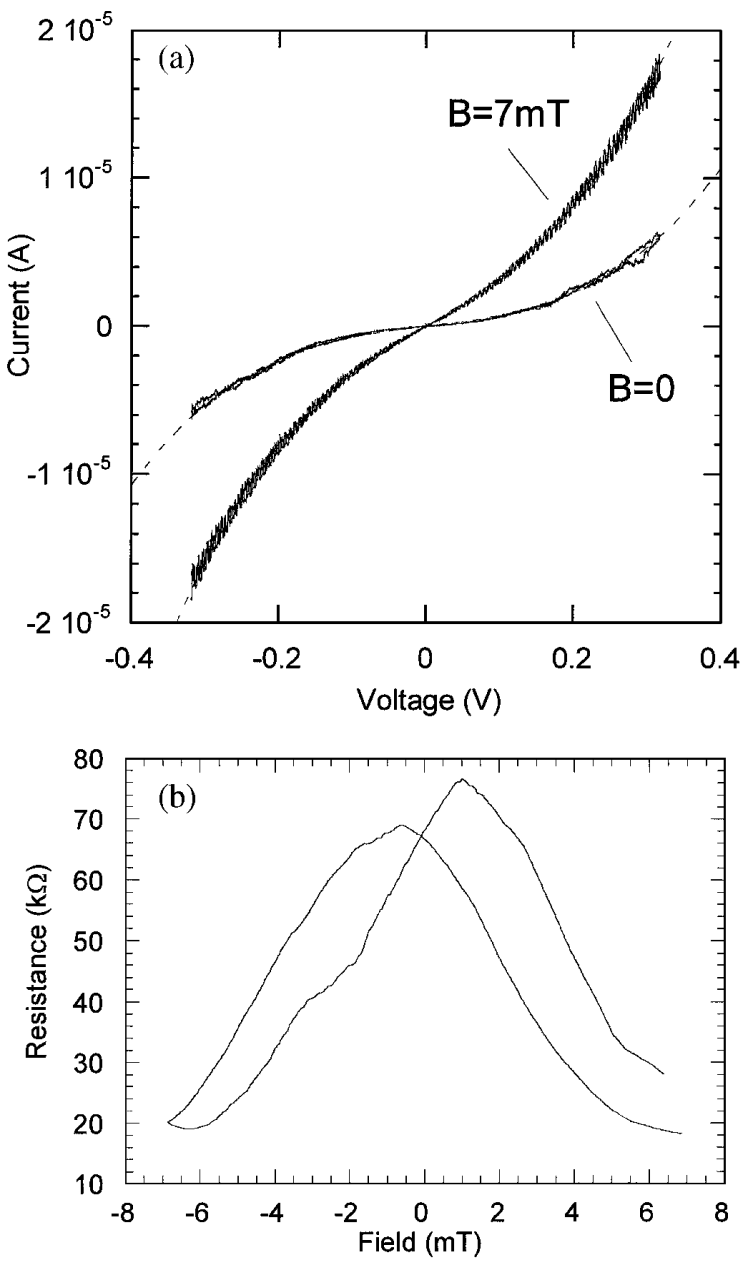

FIG. 2. Typical magnetic field effect on a nanocontact showing (a) the $I(V)$ curves with and without a field and (b) the magnetic hysteresis. nanocontacts [14]. The hysteresis proves that the magnetoresistance is related to the magnetization process of the ferrimagnetic crystallites, which, in view of their size, are certainly multidomain.

A first thought is that the effect might be due to magnetostriction. The linear magnetostriction constant $\lambda_{S}$ of $\mathrm{Fe}_{3} \mathrm{O}_{4}$ is $40 \times 10^{-6}$. The crystals used have dimensions less than $0.1 \mathrm{~mm}$, so the change of length on aligning the magnetization is $<4 \mathrm{~nm}$. Such a change of length could certainly influence the resistance of a nanocontact. Nevertheless, we discount magnetostriction as the main explanation of our observations for the following reasons.

(i) The observed magnetoresistance is always negative, regardless of the direction of the applied field. Magnetostriction would be expected to produce both positive and negative effects because the relative length change $\Delta l / l=$ $(1 / 2) \lambda_{S}\left(3 \cos ^{2} \theta-1\right)$ can be of either sign depending on the angle $\theta$ between the magnetization and the measurement direction.

(ii) No change of resistance is observed in the rotating field. Specifically, the component at $2 f$ is no more than $5 \%$ of the resistance change due to the static field.

(iii) In any case, there is no magnetorestrictive length change between configurations where the moments are aligned parallel and antiparallel.

In the demagnetized, multidomain state there is likely to be a domain wall at the contact. The energy cost of creating the wall is small because of the tiny contact area.

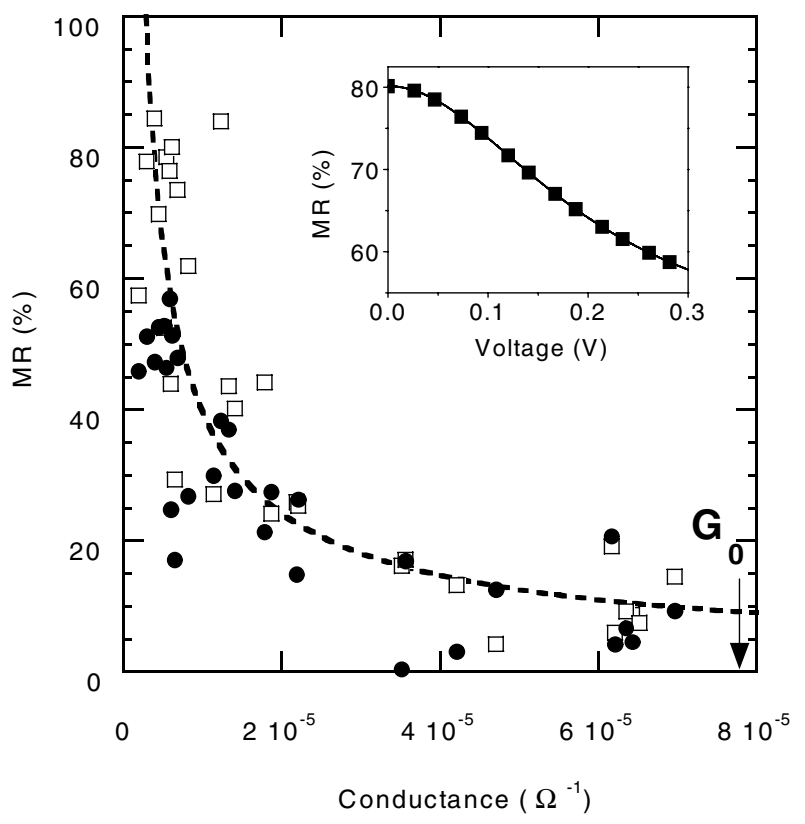

FIG. 3. Magnetoresistance data on 30 nanocontacts, with $G<$ $G_{0}$ plotted as a function of the conductance at zero bias. The solid points refer to data at $V=0.3 \mathrm{~V}$ and the open points at $V=0$, taken from the same $I(V)$ curve. The inset shows the bias dependence of the magnetoresistance of a typical contact. Note that the magnetoresistance here is defined as $[R(0)-$ $R(H)] / R(0)$ and $R=d V / d I$. 
It is known from analytical calculations [15] and Monte Carlo simulations [16] that the wall width is comparable to the size of the constriction. Furthermore, the energy per unit area of a geometrically constricted wall, of width $\delta$ much less than the natural wall width $\delta_{0}=2 \sqrt{A / K}$, is

$$
\gamma_{W} \approx \pi^{2} A / \delta .
$$

Here $A$ is the exchange stiffness $\left(\approx 10^{-11} \mathrm{~J} \mathrm{~m}^{-1}\right)$ and $K$ is the anisotropy constant. The wall width is approximately $\sqrt{S_{0}}[15,16]$, where $S_{0}$ is the cross section of the constriction. Such a narrow Néel wall is illustrated in Fig. 4(a).

The conduction of the magnetite nanocontact will be by hopping or by tunneling. It is not ballistic because $G<$ $G_{0}$, and there is little evidence for quantized conductance in the break histogram. As the electrons hop through the nanocontact, their spin is projected along the local magnetization direction of any $B$-site ions they visit along the way. In the case of a $180^{\circ}$ wall, when the hopping distance $\lambda$ is comparable to $\delta$, a spin-polarized incident electron will be projected with roughly equal probability

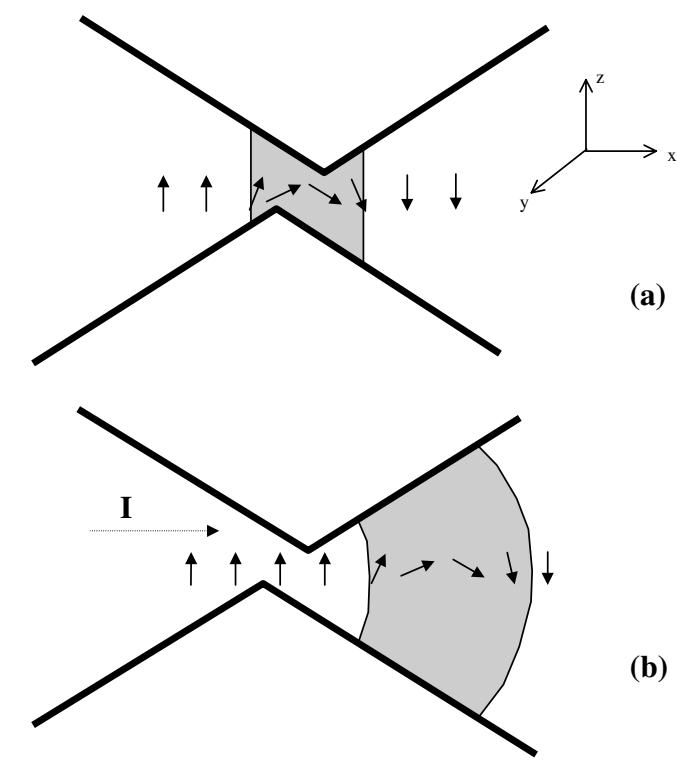

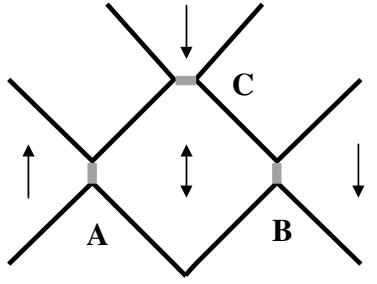

(c)

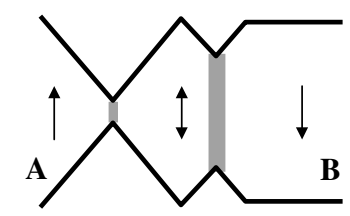

(d)
FIG. 4. (a) Sketch of a narrow domain wall constricted in a nanocontact. (b) Spin pressure pushes the domain wall into the adjacent electrode. (c) Magnetic switch based on currentinduced toggling of a domain wall between two positions $A$ and $B$. Readout is obtained by sensing the resistance across $C$. (d) Two-terminal magnetic switch (the peanut device). Resistance between terminals $A$ and $B$ depends on the location of the domain wall. into the $\uparrow$ or $\downarrow$ channels. The $\uparrow$ electrons are transmitted, while the $\downarrow$ electrons are reflected. As the directions of magnetization of the crystallites on either side of the contact are aligned, the domain wall is eliminated and the conductance increases markedly. If $G_{\mathrm{P}}^{\dagger(\downarrow)}$ and $G_{\mathrm{AP}}^{\dagger(\downarrow)}$ are the conductances of the contact in a parallel or antiparallel configuration for $\uparrow$ or $\downarrow$ electrons, then the conductance doubles in this case and the saturation magnetoresistance $\Delta R / R(0)=\left(G_{\mathrm{P}}^{\downarrow}-G_{\mathrm{AP}}^{\downarrow}\right) / G_{\mathrm{P}}^{\downarrow}=50 \%$, since $G_{\mathrm{AP}}^{\downarrow}=G_{\mathrm{P}}^{\downarrow} / 2$. More generally, the transmission probability between sites misaligned by an angle $\theta$ varies as $\cos ^{2} \theta / 2$. When $\lambda<\delta$, the transmission $T$ across the entire wall is given by

$$
T=\frac{1}{2}\left\{\cos ^{2}(\pi / 2 \nu)\right\}^{\nu-1},
$$

where $\nu=\delta / \lambda$. The magnetoresistance is $(1-T)$. The data points in Fig. 3 are distributed between $10 \%$ and $90 \%$. Values smaller than $50 \%$ may be explained by domain walls of less than $180^{\circ}$ or $\lambda<\delta$. Greater values occur when $\lambda>\delta$.

The current density in the nanocontact reaches quite high values. For example, a current of $10 \mu \mathrm{A}$ in a contact area of $1(\mathrm{~nm})^{2}$ corresponds to $j=10^{13} \mathrm{~A} \mathrm{~m}^{-2}$, and an electron drift velocity of $4600 \mathrm{~m} \mathrm{~s}^{-1}$. The rate of flow of electrons through the contact is $I / e$, which for $I=10 \mu \mathrm{A}$ corresponds to $6 \times 10^{13}$ electrons/second. The associated angular momentum, $I \hbar / 2 e$, has to be reversed as the electrons traverse the domain wall. The flow of momentum and angular momentum into the wall associated with the electron mass and spin results in an electron pressure $P_{\mathrm{el}}$. The threshold pressure needed to displace the wall from the contact, resulting in the "magnetic balloon effect" illustrated in Fig. 4(b), can be estimated from Eq. (1). It is $P_{t}=\pi^{3} \mathrm{Am} / 2 \mathrm{~S}$, where $m$ is the tangent of the half angle of the contact, modeled as a truncated cone. The pressure due to electron momentum, assuming a reflection coefficient $\beta(<1)$, is $2 \beta m_{e} j^{2} / n e^{2}$, where $m_{e}$ is the electron mass and $n=1.35 \times 10^{28} \mathrm{~m}^{-3}$ is the conduction electron density in magnetite. In the numerical example, the pressure due to the electron momentum cannot exceed $10^{6} \mathrm{~N} \mathrm{~m}^{-2}$, whereas $P_{t}=2 \times 10^{8} \mathrm{~N} \mathrm{~m}^{-2}$.

The spin pressure is related to the exchange drag effect, discussed by Berger for Permalloy films [17]. Electrons hopping onto a core spin in the wall aligned at $\psi$ to their quantization direction find their energy increased by $\Delta(1-\cos \psi) / 2$, where $\Delta$ is the energy of the first available $\uparrow$ state relative to the Fermi energy which is at the bottom of the empty $A$-site $e$ band. This leads to a pressure of order $P_{d} \approx j \tau_{\mathrm{sr}} \Delta / e \delta$, where $\tau_{\mathrm{sr}}$ is the spin relaxation time. Taking $\Delta=0.5 \mathrm{eV}[6]$ and $\tau_{\mathrm{sr}} \sim 10^{-12}-10^{-13} \mathrm{~s}$, corresponding to a typical spin wave frequency, leads to $P_{d} \approx 2 \times 10^{9} \mathrm{~N} \mathrm{~m}^{-2}$.

We model the contact as two truncated cones, with minimum radius $y_{0}$. Equating $S P_{d}$ to the force exerted by the wall $\left(d E_{w} / d x\right)$, where the wall area $S=\pi\left(y_{0}+m x\right)^{2}$, and $E_{w}=S \gamma_{w}$, gives an expression for the wall width $\delta=2 I \tau_{\mathrm{sr}} \Delta / \pi^{3} \mathrm{Aem}$, and hence the displacement 


$$
x \approx\left(I \tau_{\mathrm{sr}} \Delta / \pi^{3} A e\right)-y_{0},
$$

assuming $m \approx 1$. Inserting typical numerical values with $I=10 \mu$ A gives $x \sim 1-15 \mathrm{~nm}$. Hence, the currents used in the experiments are capable of moving the wall. Spin accumulation is unlikely to play any role in a half metal, because any local buildup of spin polarization must be accompanied by a corresponding accumulation of charge.

Previous evidence for current-induced domain wall movement has been reported in Permalloy thin films [18], nickel nanowires [19,20], and cobalt nanowires [21]. Recent results show current-induced magnetic switching in multilayers [22].

In our experiment we see evidence of electron pressure on the domain wall in two ways. One is the nonlinearity of the $I(V)$ curves. As the wall is moved away from the nanocontact it broadens and the magnetoresistance falls. Furthermore, the nonlinearity is greatly reduced when the domain wall is eliminated (Fig. 2). The second is the observation of asymmetric $I(V)$ curves in a number of cases.

Tunneling can also be invoked to explain nonlinear $I(V)$ characteristics, and it is quite possible that the atomic states of some monolayers at the surface in the nanocontact are localized, forming a tunnel barrier. Fitting the $I(V)$ characteristics to the Simmons model [23] gives barrier heights of about $0.1 \mathrm{~V}$ and widths of $1 \mathrm{~nm}$ for an area of $10 \mathrm{~nm}^{2}$. However, tunneling does not explain why the shape of the $I(V)$ characteristics changes in the small applied field. The tunnel barrier height and width should remain unchanged, with only the tunnel probability being affected by relative spin orientation across the contact.

Another source of nonlinearity arises when the carriers are polarons, as they are in magnetite: the number of phonons associated with the carriers decreases with increasing voltage [24], but again no magnetic field effect is expected. The nonlinearity generally found in metallic nanocontacts [25] is not known to be field dependent.

The magnetic balloon effect in nanocontacts may be exploited in a current-activated magnetic switch. If the magnetic material has a suitable distribution of pinning sites or if it is appropriately constricted, as suggested in Fig. 4(c) or 4(d), a region of reversible magnetization may be stabilized between the constrictions. Switching in the "peanut" device can be very fast, as the wall moves only about $10 \mathrm{~nm}$, at a velocity of order $100 \mathrm{~m} \mathrm{~s}^{-1}$ [26]. In Fig. 4(c), a third conact is required for readout, but Fig. 4(d) is a two-terminal device with high and low resistance states for the $\uparrow$ and $\downarrow$ configurations of the central region. This could be the basis of a new magnetic nanocontact memory architecture with exceptionally efficient magnetoresistive readout.

This work was supported by the EU as part of the AMORE project in the growth program, and by Enterprise Ireland under Contract No. SC/99/225.

[1] Yu Lu et al., Phys. Rev. B 54, R8357 (1996).

[2] J. M. de Teresa et al., Phys. Rev. Lett. 82, 4288 (1999).

[3] H. Y. Hwang, S-W. Cheong, N. P. Ong, and B. Batlogg, Phys. Rev. Lett. 77, 2041 (1996).

[4] J. M. D. Coey et al., Phys. Rev. Lett. 80, 3815 (1998).

[5] M. Pénicaud, B. Siberchicot, C. B. Sommers, and J. Kubler, J. Magn. Magn. Mater. 103, 212 (1992).

[6] V.A. M. Brabers, in Handbook of Magnetic Materials, edited by K. H. J. Buschow (North-Holland, Amsterdam, 1998), Vol. 8, p. 189.

[7] Crystals were grown by Crystalab, Sofia.

[8] The device uses a PI P-841.60 piezo stack and power supply. It is vibration isolated using a stack of six $22 \mathrm{~cm}$ MFD plates separated by $150 \mathrm{~mm}$ diameter neoprene O-rings, resting on a metal plate supported by a bicycle inner tube.

[9] J. L. Costa-Krämer et al., Phys. Rev. B 55, 5416 (1997).

[10] F. Ott et al., Phys. Rev. B 58, 4656 (1998).

[11] J. J. Versluijs et al., J. Magn. Magn. Mater. 211, 212 (2000).

[12] M. Viret et al., Europhys. Lett. 39, 545 (1997).

[13] S. S. P. Parkin et al., J. Appl. Phys. 85, 5828 (1999).

[14] N. Garcia, M. Munoz, and Y.-W. Zhao, Phys. Rev. Lett. 82, 2923 (1999).

[15] P. Bruno, Phys. Rev. Lett. 83, 2425 (1999).

[16] Y. Labaye, L. Berger, and J. M. D. Coey (to be published).

[17] L. Berger, J. Appl. Phys. 55, 1954 (1984).

[18] P. P. Freitas and L. Berger, J. Appl. Phys. 57, 1266 (1985).

[19] J.-E. Wegrowe et al., Europhys. Lett. 45, 626 (1999).

[20] N. Garcia, H. Rohrer, I. G. Saveliev, and Y.-W. Zhao, Phys. Rev. Lett. 85, 3053 (2000).

[21] U. Ebels et al., Phys. Rev. Lett. 84, 983 (2000).

[22] E. B. Myers et al., Science 285, 867 (1999).

[23] J. G. Simmons, J. Appl. Phys. 34, 238 (1963).

[24] F. Guinea (private communication).

[25] J. I. Pascual et al., Science 267, 1793 (1995).

[26] A. Hubert and R. Schäfer, Magnetic Domains (SpringerVerlag, Berlin, 1998). 